\title{
Tumor Maligno de la Vaina del Nervio Periférico del Mediastino en un Paciente con Neurofibromatosis Tipo 1
}

\author{
Mediastinal Malignant Peripheral Nerve Sheath Tumor in a Neurofibromatosis Type 1 Patient
}

*Julio Alexander Diaz-Perez \& **Edgar Julian Ferreira Bohorquez

DIAZ-PEREZ, J. A. \& FERREIRA, B. E. J. Tumor maligno de la vaina del nervio periférico del mediastino en un paciente con neurofibromatosis tipo 1. Int. J. Morphol., 29(1):133-139, 2011.

RESUMEN: El tumor maligno de la vaina del nervio periférico (TMVNP), es una neoplasia maligna originada en las células de Schwan de la vaina de revestimiento del nervio periférico. Describir el caso de un hombre con neurofibromatosis tipo 1 (NF1), quién presentó un TMVNP de bajo grado, y realizar una discusión sobre esta enfermedad. Hombre de 28 años, con antecedente de NF1 diagnosticada a los 15 años de edad, con dolor pleurítico izquierdo, disnea y pérdida de peso de 10 meses de evolución. Al examen de tórax, se observó marcada hipercifosis dorsal con disminución del murmullo pulmonar. La radiografía de tórax y tomografía axial computarizada (TAC), evidenciaron gran masa radioopaca bien delimitada en mediastino posterior. Por lo anterior, se realizo biopsia por punción con aguja gruesa guiada por TAC, en la cual se identificó una neoplasia maligna mesenquimal. Se decidió realizar resección del tumor a través de toracotomía posterolateral, en la que se obtuvo gran masa de 8x9x9 cm, de superficie externa irregular, pardo-violácea y consistencia firme. El estudio histopatologico e inmunofenotípico concluyo el diagnóstico de TMVNP en mediastino posterior Grado 1. Posterior a la cirugía, el paciente se encuentra asintomático. Se presentó un caso de TMVNP originado en un paciente con NF1, presentación que generalmente cursa con peor pronóstico, además se realizo una breve revisión de los aspectos más relevantes de esta enfermedad, algunos de los cuales han tenido un avance vertiginoso en años recientes.

PALABRAS CLAVE: Tumor maligno de la vaina del nervio periferico; Neoplasias de la vaina del nervio; Neurofibromatosis 1; Neoplasias del mediastino.

\section{INTRODUCCIÓN}

El tumor maligno de la vaina del nervio periférico (TMVNP), también denominado schwanoma maligno, sarcoma neurogénico, neurofibrosarcoma, neurilemoma maligno y sarcoma neurogénico, es una neoplasia maligna originada de las células de Schwan de la vaina del revestimiento de los nervios periféricos (Enzinger \& Weiss, 1995; Woodruff et al., 2000). Se localiza con mayor frecuencia en las partes profundas de la región de la cabeza y el cuello, columna y extremidades inferiores (Enzinger \& Weiss; Woodruff et al.; Minovi et al., 2007). Esta lesión, es la responsable de alrededor del 5\% de los sarcomas de partes blandas, con una incidencia de 1 caso por millón de personas año (Woodruff et al.; Anghileri et al., 2006). La razón hombre: mujer es de 1:1 y la edad típica de presentación se encuentra en un amplio rango (desde los 20 hasta los 50 años de edad), observándose aproximadamente
10 años antes en pacientes con neurofibromatosis tipo 1 (NF1) (Enzinger \& Weiss; Woodruff et al.). Estas lesiones se han asociado a diferentes factores, siendo el más implicado (hasta en el 50\% de los casos), la NF1 (Enzinger \& Weiss; Woodruff et al.; Anghileri et al., 2006); esta última es una enfermedad en la que se originan múltiples neurofibromas, algunos de los cuales con capacidad de transformación a un TMVNP, entre los que se destacan los neurofibromas plexiformes que presentan un mayor riesgo de progresión a la malignidad (Grobmyer et al., 2008; Korf, 2000).

El desarrollo de los TMVNP también se ha asociado a la exposición a radiación, presentándose desde 4 a 40 años después de la misma, y a la presencia de ganglioneuromas (Enzinger \& Weiss; Woodruff et al.; 
Grobmyer et al.). Sin embargo, en el 50\% de los casos no se encuentran características asociadas (Grobmyer et al.; Korf). Esta enfermedad tiene diferentes características, entre las que se encuentran su capacidad de recurrencia locoregional y de metástasis a distancia. Por otro lado, se han realizado múltiples avances en el conocimiento de su etiología, características patológicas, imagenología y tratamiento. Por lo anterior, se describe el caso de un hombre con NF1, quién presentó un TMVNP de bajo grado, y se realiza una discusión sobre esta neoplasia.

\section{CASO CLÍNICO}

Hombre de 28 años de edad, con dolor pleurítico en hemotórax izquierdo, disnea y pérdida de peso de 10 meses de evolución, que aumentó progresivamente en intensidad. El paciente tiene antecedente de NF1 diagnosticada a los 15 años de edad y antecedente familiar de padre y hermano con NF1. Al examen físico no presentaba alteraciones en sus signos vitales, ni en cabeza y cuello. En el tórax, se encontró marcada hipercifosis dorsal con disminución del murmullo vesicular en los 2 tercios inferiores del hemotórax izquierdo, donde se encontró además matidez a la percusión, no se auscultaron ruidos agregados. El examen de abdomen y extremidades fue normal. A nivel neurológico se evidenció miosis en pupila izquierda con ptosis palpebral ipsilateral. En piel presentaba gran cantidad de manchas café con leche, entre 1 y $8 \mathrm{~cm}$ de diámetro, distribuidas en tronco y extremidades, además de masas subcutáneas firmes distribuidas en forma generalizada con diámetros variables de 0,5 a $2 \mathrm{~cm}$. En la ra-

diografía de tórax, se evidenció gran masa radioopaca bien delimitada en mediastino posterior, que desplazaba las estructuras cardiovasculares a la región anterior (Fig. 1). La tomografía axial computarizada (TAC) de tórax mostró gran masa en mediastino posterior lateralizada a la izquierda, de 79 x $91 \mathrm{~mm}$, con una densidad de 41 U.H, la cuál presentaba áreas de degeneración quística en su interior.

Además, se evidenció en mediastino anterosuperior derecho otra lesión de $2 \mathrm{~cm}$ de diámetro de igual densidad (Fig. 2). Por lo anterior, se decidió realizar biopsia por punción con aguja gruesa de la masa mediastinal izquierda guiada por TAC. Se obtuvo una muestra donde se identificó una neoplasia maligna mesenquimal compatible con tumor maligno de la vaina del nervio periférico, posterior a los estudios histopatológicos e inmunohistoquímicos. Se decidió realizar resección del tumor, a través de toracotomía posterolateral, en la que se obtuvo gran masa de $8 \times 9 \times 9$ $\mathrm{cm}$, de superficie externa irregular, pardo-violácea, ligeramente congestiva y de consistencia firme. Al corte, la masa presentaba una superficie heterogénea, blanco amarillenta, con múltiples áreas de degeneración quística, repletos de material mixoide, entremezclado con hemorragia (Fig. 3). En el estudio histopatológico se observó una neoplasia maligna mesenquimal compuesta por gran cantidad de células fusiformes, con núcleos aumentados de tamaño irregulares, con cromatina vesiculosa, nucleolos prominentes y ocasionales figuras mitóticas atípicas (7 en 10 campos de alto aumento) rodeadas por un citoplasma eosinófilo tenue; éstas células se disponían en forma fasicular, formando empalizadas sobre un estroma finamente colagenizado, en el que se reconocían además, es-
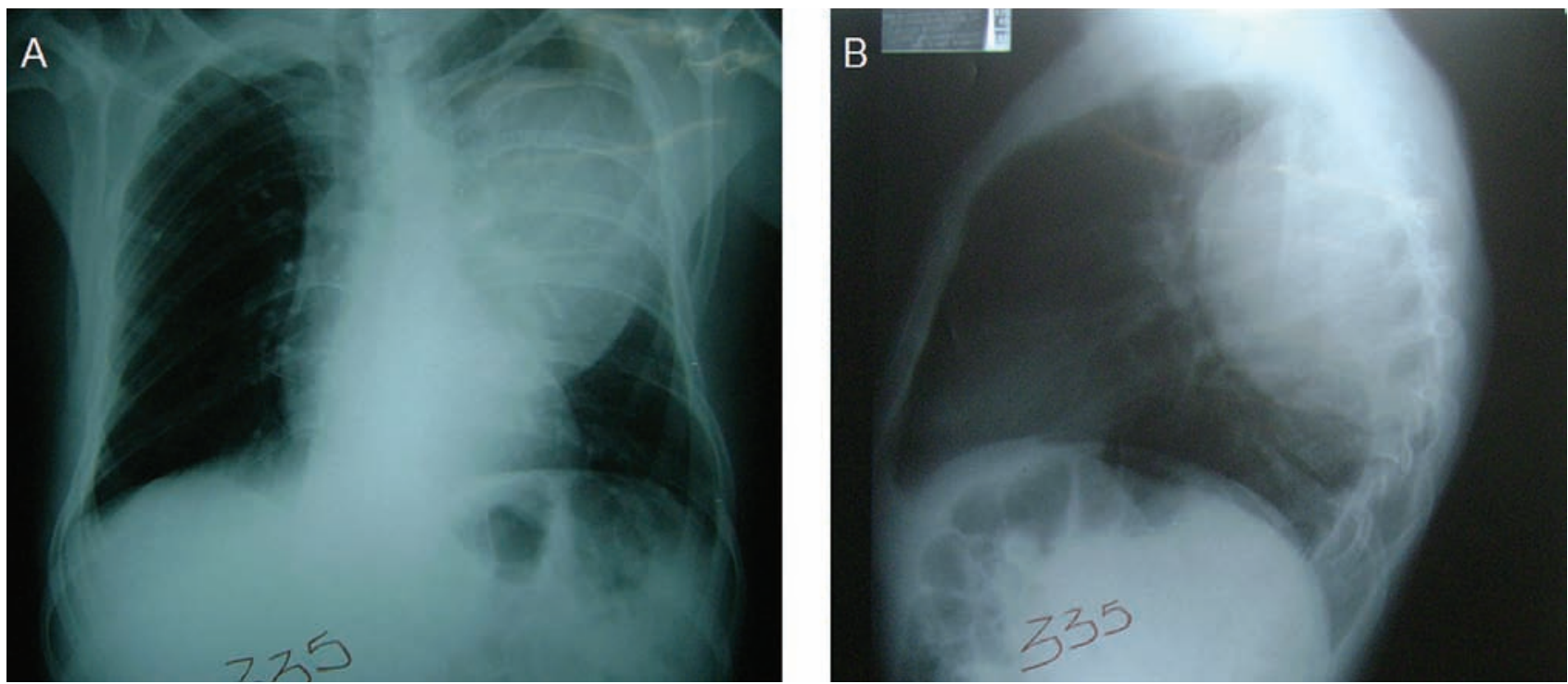

Fig. 1. Radiografía de tórax (A Postero anterior; y B Lateral) mostro gran masa radioopaca bien delimitada en mediastino posterior, que desplazaba las estructuras cardiovasculares a la región anterior. 


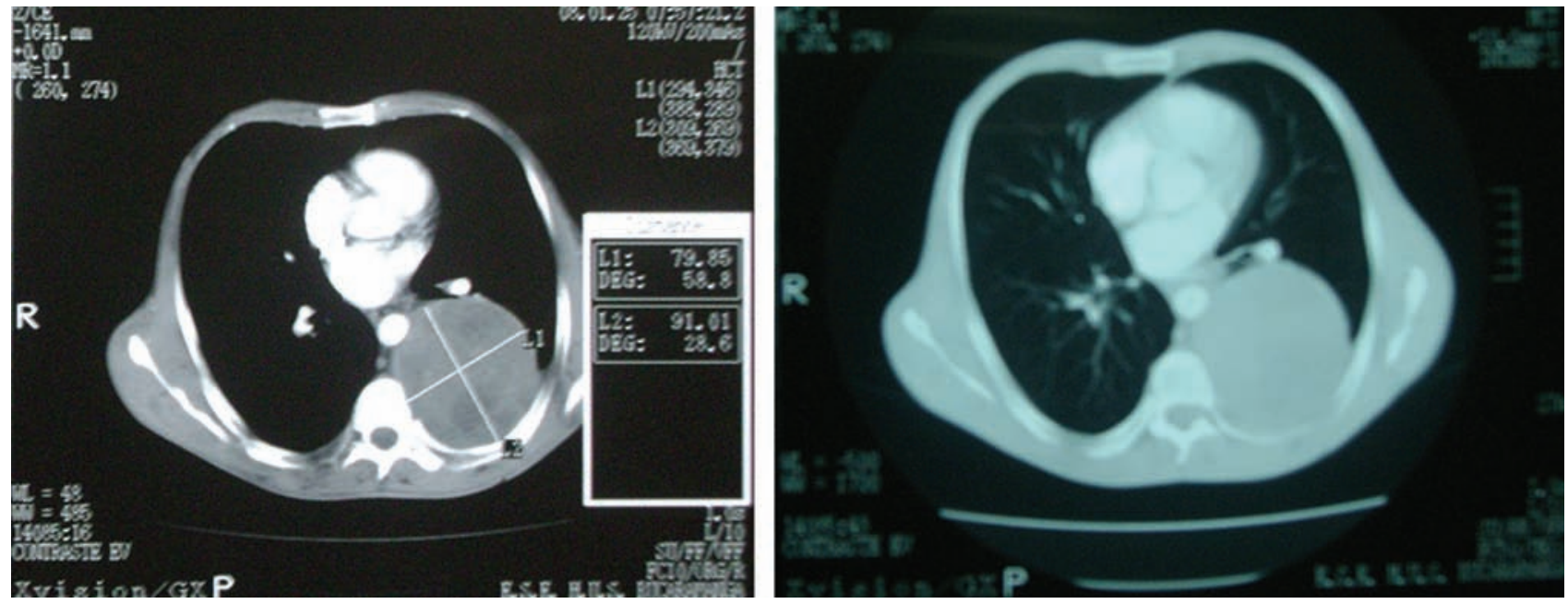

Fig. 2. Tomografía axial computarizada (TAC) de tórax (A contrastada y B simple) mostró gran masa en mediastino posterior lateralizada a la izquierda, de 79x91 mm, con densidad de 41U.H, en su interior se observaban áreas de degeneración quística. Además se evidenció en mediastino anterosuperior derecho la presencia de una pequeña lesión de $2 \mathrm{~cm}$ de diámetro de igual densidad.
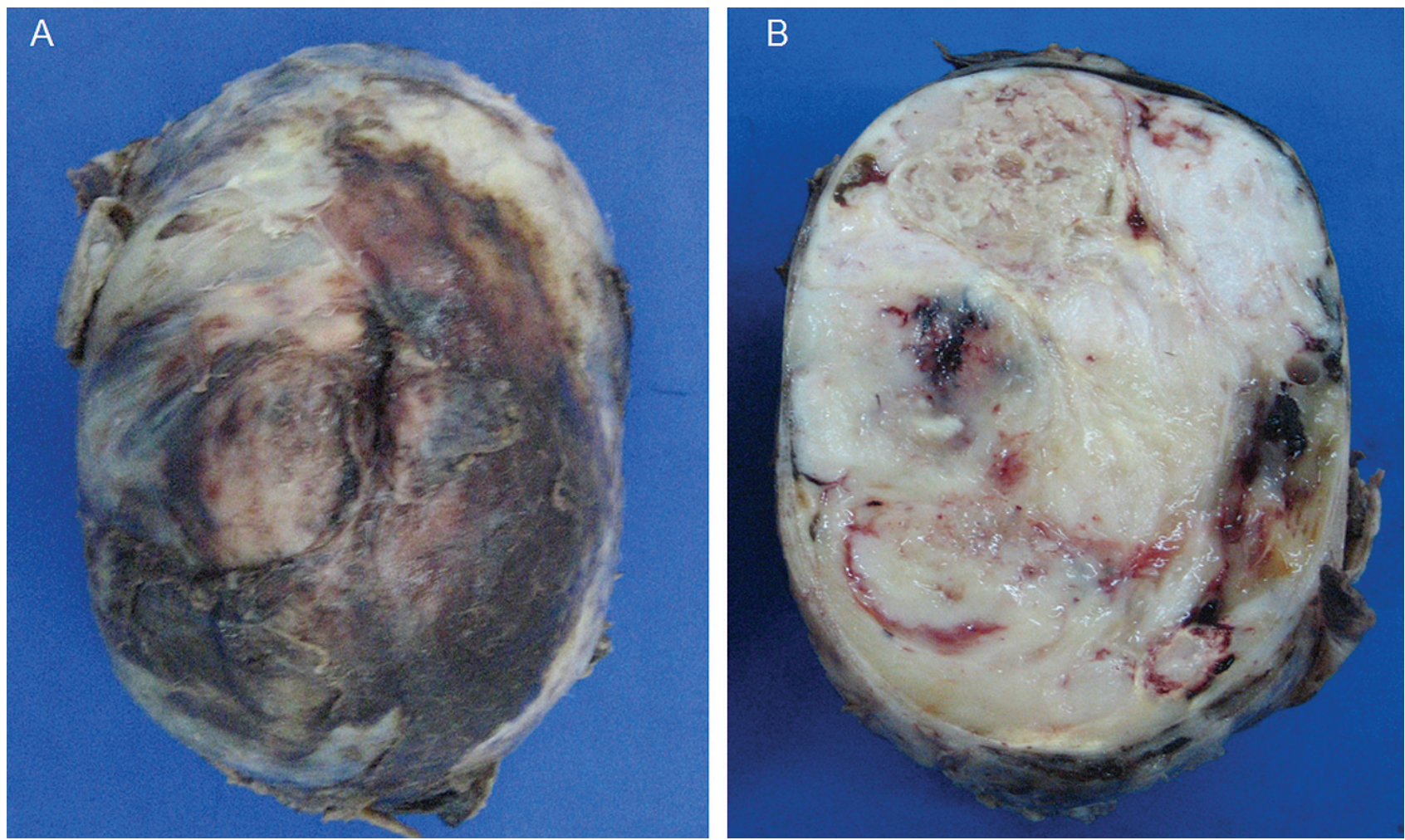

Fig. 3. Visión macroscópica del tumor del mediastino, en A se observa gran masa de 8 x 9 x 9 cm, de superficie externa irregular, pardoviolácea, congestiva y de consistencia firme; y en B se muestra la superficie de corte de la masa la cual era heterogénea, blanco amarillenta, con múltiples áreas de degeneración quística, repleta de material mixoide, entremezclada con hemorragia.

pacios pseudovasculares y áreas de necrosis geográfica (Fig. 4). Estas células tumorales presentaron positividad para CD99 y S100. Finalmente, se realizó el diagnóstico de TMVNP en mediastino posterior Grado 1. Posterior a la ci- rugía, el paciente presentó adecuada evolución en su postoperatorio, con reexpansión aceptable del pulmón izquierdo, por lo que se dio de alta y actualmente se encuentra asintomático. 

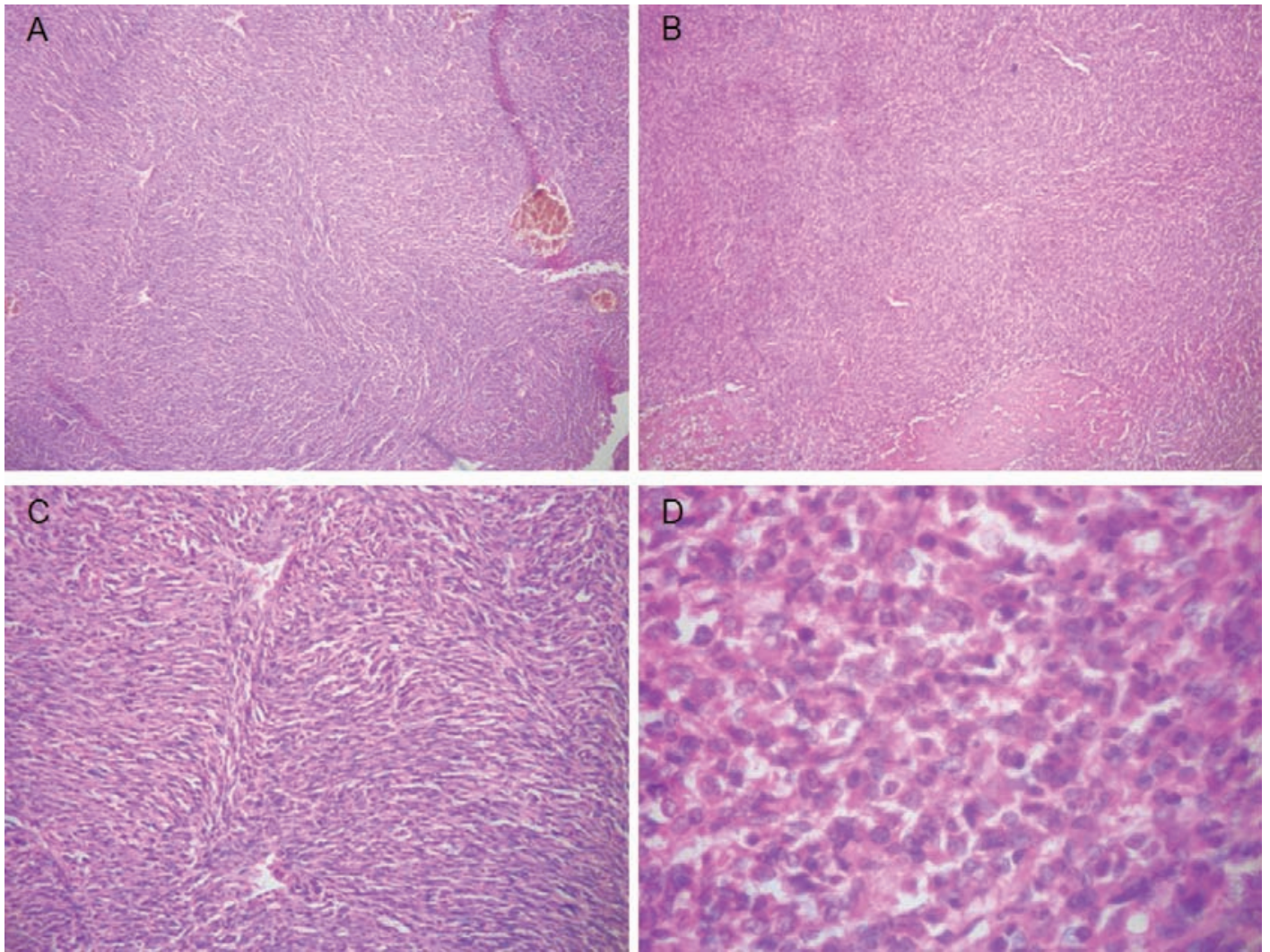

Fig. 4. En el estudio histopatológico se reconoció en A y D una neoplasia maligna mesenquimal, compuesta por células fusiformes, con núcleos aumentados de tamaño irregulares, con cromatina vesiculosa, nucleolos prominentes y ocasionales figuras mitóticas atípicas ( 7 en 10 campos de alto aumento) rodeadas por citoplasma eosinófilo tenue; éstas células se disponían en forma fasicular, formando empalizadas sobre un estroma colagenizado, en el que se reconocían además, espacios pseudovasculares y áreas de necrosis geográfica (B y C); (HE 4x,10x y 40x).

\section{DISCUSIÓN}

En años recientes, el conocimiento de la etiología del TMVNP ha avanzado vertiginosamente. Actualmente, se considera que esta neoplasia maligna, se origina posterior a alteraciones citogenéticas encontradas en los cromosomas $1,2,5,7-9,11-14,17,18$ y 22 , las cuales producen alteraciones en diferentes genes (Woodruff, 1999; Ishiguro et al., 2006; Grobmyer et al., 2008; Perrone et al., 2003), entre los cuales, el más frecuentemente lesionado es la deleción del gen NF1 (Perrone et al.; Agesen et al., 2005; Mertens et al., 2000). Las demás alteraciones encontradas son listadas en la Tabla I.

Esta enfermedad se manifiesta clínicamente con la presencia de masa que aumenta progresivamente de tamaño, acompañada de dolor, parestesias y deficit neurológico.

Se encuentra principalmente en relación con grandes nervios, como aquellos de las extremidades y de la columna vertebral (Shimizu et al., 2008). En el abordaje diag-
Tabla I. Alteraciones genéticas frecuentemente encontradas en el TMVNP (Mantripragada et al., 2008).

\begin{tabular}{lcl}
\hline Gen & $\begin{array}{c}\text { Porcentaje de casos } \\
\text { de TMVNP con } \\
\text { esta alteración }\end{array}$ & Alteración \\
\hline NF 1 & $71 \%$ & Deleción \\
HMMR/RHAMM & $46 \%$ & Deleción \\
MMP13 & $43 \%$ & Deleción \\
p16-INK4a & $39 \%$ & Deleción \\
LICAM2 & $29 \%$ & Deleción \\
RASSF2 & $25 \%$ & Deleción \\
RB1 & $25 \%$ & Deleción \\
ITGB4 & $32 \%$ & Amplificación \\
PDGFRA & $29 \%$ & Amplificación \\
BIRC5 & $25 \%$ & Amplificación \\
CCNE2 & $25 \%$ & Amplificación \\
EGFR & $25 \%$ & Amplificación \\
HGF & $25 \%$ & Amplificación \\
MET & $25 \%$ & Amplificación \\
TERT & $25 \%$ & Amplificación \\
\hline
\end{tabular}


nóstico son de importancia los estudios imagenológicos (Enzinger \& Weiss; Anghileri et al.; Carli et al., 2005). La resonancia magnética nuclear es la modalidad preferida a la hora de establecer la diferenciación entre un tumor de origen neurogénico Vs no neurogénico. Sin embargo, la TAC es de gran utilidad en la evaluación de aquellos tumores ubicados en mediastino y retroperitoneo, siendo de elección para evaluar el compromiso de hígado y pulmón (Anghileri et al.).

En los últimos años, métodos de diagnóstico mínimamente invasivos, como la citología por punción con aguja fina y la biopsia por punción con aguja gruesa han ganado gran relevancia en el estudio prequirúrgico del TMVNP. En la citología se obtienen células fusiformes, de contornos irregulares, que muestran diferenciación neural, las cuales se distribuyen en fascículos sobre un estroma fibrilar. En estas células se puede observar ligera irregularidad nuclear y ocasionales mitosis (McGee et al., 1997; Jiménez-Heffernan et al., 1999). Al complementar el estudio con inmunocitoquímica, se puede mejorar su rendimiento diagnóstico (Molina et al., 2001). Por otro lado, en las muestras obtenidas por punción con aguja gruesa, se observan lesiones marcadamente celulares con diferenciación neural, acompañadas de mitosis frecuentes y áreas de necrosis geográfica (Molina et al.). Al complementar este exámen diagnóstico con tinciones inmunohistoquímicas como el S100, CD 34, MIB1, TOPOIIA y P53, se obtiene un gran rendimiento diagnóstico (McGee $e t$ al.; Jiménez-Heffernan et al.; Molina et al.).

En el estudio patológico final estas neoplasias se caracterizan macroscópicamente por ser grandes masas regulares localizadas adyacentes a un nervio, de consistencia firme, que se disponen en forma fusiforme y que presentan múltiples áreas de degeneración quística y mixoide (Enzinger \& Weiss; Woodruff et al.; Woodruff, 1999; Scheithauer et al., 2000). En el estudio histopatológico, se reconoce gran cantidad de células monomórficas dispuestas en fascículos, conformando ocasionales empalizadas y espacios pseudovasculares. Las células que conforman esta lesión tienen la particularidad de presentar mitosis frecuentes y occasional morfología bizarra. Además, se encuentran células tumorales distribuidas en forma perivascular. Otros hallazgos relevantes son la presencia de necrosis geográfica y de metaplasia mesenquimal, observada en el $15 \%$ de los casos, que puede ser condroide, ósea o muscular (Enzinger \& Weiss; Woodruff et al.; Scheithauer et al., 2000). En muy pocos casos, se reconoce también diferenciación glandular y pigmentación con melanina (Woodruff, 1976). En el estudio inmunohistoquímico sus células son positivas para CD99 O13 en el 86\% de los casos, S100 en el 62\%, CD57 en el $55 \%$, colágeno tipo IV, producto de la proteína genética 9,5 y p53 y son negativos para antígeno de membrana epitelial, citoqueratinas y CD19 (Zhou et al., 2003; Olsen et al., 2006).
En el estudio por microscopía electrónica se observa plegamiento de la membrana celular, con configuración lamelar, presencia de membrana basal discontínua, evidencia de uniones intercelulares y presencia de ocasionales gránulos densos (Enzinger \& Weiss; Woodruff et al., 2000; Woodruff, 1999). En la determinación del grado de estas lesions neoplásicas, se utiliza el sistema propuesto por la French Federation of Cancer Centers Sarcoma Group, que las divide en 3 grupos, teniendo en cuenta la diferenciación tumoral, conteo mitótico y necrosis tumoral: Grado 1: score total de 2-3; Grado 2: score de 4-5; Grado 3: score de 6-8 (Coindre et al., 1996). El diagnóstico diferencial del TMVNP, debe hacerse con el liposarcoma pleomórfico, histiocitoma fibroso maligno, sarcoma sinovial y lesiones neurales, de los cuales se puede diferenciar mediante su perfil inmunohistoquímico (Korf; Woodruff, 1999; Woodruff, 1976; Zhou et al.; Olsen et al.).

Algunas variantes inusuales de esta lesión son la variante epitelioide y el tumor maligno de Tritón. En la variante epitelioide, que correponde al 5\% de los TMVNP, se observa una lesión neoplásica maligna de origen mesenquimal compuesta por células epitelioides con un citoplasma acidofílico; esta variante es la más frecuentemente vista en transformación maligna de los neurofibromas no asociados con neurofribromatosis; a nivel de inmuno-histoquímica, esta lesión presenta reacción a HMB45, por lo que se debe plantear el diagnóstico diferencial con un melanoma desmoplástico, para lo que es de gran ayuda la positividad inmunofenotípica que muestra esta lesión para el producto del gen 9.5 y la negatividad para la proteína S100 (Lodding et al., 1986). Por otro lado, el tumor maligno de Tritón, o TMVNP con diferenciación rabdomio-sarcomatosa, descrito inicialmente por Masson en 1932, fue denominado así debido a que puede ser inducido al contacto por las salamandras tipo Tritón; trabajo realizado por Locatelly en 1925 (citados por Stasik \& Tawfik, 2006). En 1972 Woodruff (Woodruff et al., 1973; Rekhi et al., 2008) estableció 3 criterios para su diagnóstico, que son: 1. Que el tumor se origine en el nervio periférico, en un ganglioneuroma, o en un paciente con neurofibromatosis tipo 1. 2. Que el tumor tenga características de crecimiento propias de las células de Schwann. 3. Que existan rabdomioblastos presentes en el tumor.

El tratamiento de esta enfermedad es netamente quirúrgico planteándose como meta la resección con márgenes histopatológicos libres entre 3 y $5 \mathrm{~cm}$, es de gran importancia resaltar que en ocasiones puede requerirse resecciones en bloque de la pared toráxica que pueden llevar a alteraciones funcionales de la misma (Anghileri et al.; Korf; Carli et al.). En neoplasias inrresecables o metastáticos se ha planteado la opción de la quimioterapia presentando una respuesta mas favorable en aquellos pacientes que no cursan con una neurofibromatosis tipo 1 ; el régimen terapéutico mas estudia- 
do es el de ifosfamida + doxurrubicina; la radioterapia aunque no ha demostrado mejorar la sobrevida puede llevar a control local del tumor en pacientes con la enfermedad irresecable localmente avanzada (Korf; Masui et al., 2004).

La recurrencia presenta tasas entre el 20 y el $40 \%$, presentándose entre dos meses y 10 años a partir del diagnostico aunque la mayoría se manifiesta a los 2 años, las metástasis principalmente en pulmón se presentan entre el 20 y el $40 \%$ de los casos.

Otros sitios que se pueden comprometer por metás- tasis son hígado, nodos linfáticos y cerebro (Anghileri et al.; Carli et al.). En general, la mayoría de estudios reporta una pobre sobrevida que varia entre el 22 y el $57 \%$ a 10 años, mejorando en aquellos que cursan sin neurofibromatosis tipo 1 (Korf).

En conclusión, presentamos un caso de TMVNP el cual se originó en un paciente con neurofibromatosis tipo 1, presentación que generalmente cursa con un peor pronóstico, además se realizó una breve revisión de los aspectos más relevantes de esta enfermedad, algunos de los cuales han tenido un avance vertiginoso en años recientes.

DIAZ-PEREZ, J. A. \& FERREIRA, B. E. J. Mediastinal malignant peripheral nerve sheath tumor in a neurofibromatosis type 1 patient. Int. J. Morphol., 29(1):133-139, 2011.

SUMMARY: The malignant peripheral nerve sheath tumor (MPNST) is a malignant neoplasm originated in the Schwan cells of the periferic nerves sheath. We describe a case of a man with Neurofibroatosis Type 1 (NF1), who developed a low grade MPNST, and subsequent to a discussion of this disease. 28-year-old Man with pleuritic pain in the left hemithorax, dyspnea and weight loss, with a previous diagnosis of NF1, from the age of 15 and a family history of NF1. At chest examination the patient had an intense thoracic kyphosis, with a decline in the ventilation of the inferior two thirds of the left hemithorax, where a dull sound to percusión was also found. The chest $\mathrm{X}$ rays showed a large radiopaque and well delimited mass in the posterior mediastinum, that pushed the cardiovascular structures to the anterior region, which was also documented by chest computed tomography (CT). In view of the above, a puncture biopsy was performed with thick needle guided by CT, from where a malignant mesenhymal neoplasm was identified. It was decided to perform the resection of the tumor of the left posterior mediastinum, by left posterior lateral thoracotomy, in which a large mass of 8x9x 9 $\mathrm{cm}$ was obtanied, with irregular external surface, brown-violet, and firm. The histopathological and inmunophenotypic study concluded the diagnosis of MPNST in the posterior mediastinum grade 1. Following surgery the patient was asymptomatic. We present a case of MPNST which originated in a patient with NF1, who would usually have a worse prognosis. A brief review of the more relevant aspects of this disease was also reported, some of which have shown important progress in recent years.

KEY WORDS: Malignant peripheral nerve sheath tumor; Nerve Sheath Neoplasms; Neurofibromatosis 1; Mediastinal neoplasms.

\section{REFERENCIAS BIBLIOGRÁFICAS}

Agesen, T. H.; Flørenes, V. A.; Molenaar, W. M.; Lind, G. E.; Berner, J. M.; Plaat, B. E.; et al. Expression patterns of cell cycle components in sporadic and neurofibromatosis type 1-related malignant peripheral nerve sheath tumors. J. Neuropathol. Exp. Neurol., 64:74-81, 2005.

Anghileri, M.; Miceli, R.; Fiore, M.; Mariani, L.; Ferrari, A.; Mussi, C., et al. Malignant peripheral nerve sheath tumors: Prognostic factors and survival in a series of patients treated at a single institution. Cancer, 107:1065-74, 2006.

Carli, M.; Ferrari, A.; Mattke, A.; Zanetti, I.; Casanova, M.; Bisogno, G.; et al. Pediatric malignant peripheral nerve sheath tumor: The Italian and German soft tissue sarcoma cooperative group. J. Clin. Oncol., 23:8422-30, 2005.

Coindre, J. M.; Terrier, P.; Bui, N. B.; Bonichon, F.; Collin, F.; Le Doussal, V.; et al. Prognostic factors in adult patients with locally controlled soft tissue sarcoma. A study of 546 patients from the French Federation of Cancer Centers Sarcoma Group. J. Clin. Oncol., 14:869-77, 1996.

Enzinger, F. M. \& Weiss, S. W. Malignant tumors of peripheral nerves. In: Enzinger, F. M. \& Weiss, S. W. (Eds.) Soft tissue tumors. St. Louis, C. V. Mosby, 1995. p.889-928.

Grobmyer, S. R.; Reith, J. D.; Shahlaee, A.; Bush, C. H. \& Hochwald, S. N. Malignant peripheral nerve sheath tumor: molecular pathogenesis and current management considerations. J. Surg. Oncol., 97:340-9, 2008.

Ishiguro, M.; Iwasaki, H.; Takeshita, M.; Hirose, Y. \& Kaneko, Y. A cytogenetic analysis in two cases of malignant peripheral nerve sheath tumor showing hypodiploid karyotype. Oncol. Rep., 16:225-32, 2006. 
Jiménez-Heffernan, J. A.; López-Ferrer, P.; Vicandi, B.; Hardisson, D.; Gamallo, C. \& Viguer, J. M. Cytologic features of malignant peripheral nerve sheath tumor. Acta Cytol., 43:175-83, 1999.

Korf, B. R. Malignancy in Neurofibromatosis type 1. Oncologist, 5:477-85, 2000.

Lodding, P.; Kindblom, L. G. \& Angervall, L. Epithelioid malignant schwannoma: a study of 14 cases. Virchows Arch. A Pathol. Anat. Histopathol., 409:433-51, 1986.

Masui, F.; Yokoyama, R.; Soshi, S.; Beppu, Y.; Asanuma, K. \& Fujii, K. A malignant peripheral nerve sheath tumor responding to chemotherapy. J. Bone Joint Surg. Br., 86:113-5, 2004.

Mantripragada, K. K.; Spurlock, G.; Kluwe, L.; Chuzhanova, N.; Ferner, R. E.; Frayling, I. M.; et al. High-resolution DNA copy number profiling of malignant peripheral nerve sheath tumors using targeted microarray-based comparative genomic hybridization. Clin. Cancer Res., 14:1015-24, 2008.

McGee, R. S. Jr.; Ward, W. G. \& Kilpatrick, S. E. Malignant peripheral nerve sheath tumor: a fine-needle aspiration biopsy study. Diagn. Cytopathol., 17:298-305, 1997.

Mertens, F.; Dal Cin, P.; De Wever, I.; Fletcher, C. D.; Mandahl, N.; Mitelman, F; et al. Cytogenetic characterization of peripheral nerve sheath tumours: a report of theCHAMP study group. J. Pathol., 190:31-8, 2000.

Minovi, A.; Basten, O.; Hunter, B.; Draf, W. \& Bockmühl, U. Malignant peripheral nerve sheath tumors of the head and neck: management of 10 cases and literature review. Head Neck, 29:439-45, 2007.

Molina, C. P.; Putegnat, B. B. \& Logroño, R. Fine-needle aspiration cytology and core biopsy of malignant peripheral nerve sheath tumor of the uterus: a case report. Diagn. Cytopathol., 24:347-51, 2001.

Olsen, S. H.; Thomas, D. G. \& Lucas, D. R. Cluster analysis of immunohistochemical profiles in synovial sarcoma, malignant peripheral nerve sheath tumor, and Ewing sarcoma. Mod. Pathol., 19:659-68, 2006.

Perrone, F.; Tabano, S.; Colombo, F.; Dagrada, G.; Birindelli, S.; Gronchi, A.; et al. p15INK4b, p14ARF, and p16INK4a inactivation in sporadic and neurofibromatosis type 1-related malignant peripheral nerve sheath tumors. Clin. Cancer Res., 9:4132-8, 2003.
Rekhi, B.; Jambhekar, N. A.; Puri, A.; Agrawal, M. \& Chinoy, R. Clinicomorphologic features of a series of 10 cases of malignant triton tumors diagnosed over 10 years at a tertiary cancer hospital in Mumbai, India. Ann. Diagn. Pathol., 12:90-7, 2008.

Scheithauer, B. W.; Woodruff, J. M. \& Erlandson, R. A. Primary malignant tumors of the peripheral nerve. In: Tumors of the Peripheral Nervous System. Washington, DC: Armed Forces Institute of Pathology, 2000. pp.336-40.

Shimizu, J.; Arano, Y.; Murata, T.; Ishikawa, N.; Yachi, T.; Nomura, T.; et al. A case of intrathoracic giant malignant peripheral nerve sheath tumor in neurofibromatosis type I (von Recklinghausen's disease). Ann. Thorac. Cardiovasc. Surg., 14:42-7, 2008.

Stasik, C. J. \& Tawfik, O. Malignant peripheral nerve sheath tumor with rhabdomyosarcomatous differentiation (malignant triton tumor). Arch. Pathol. Lab. Med., 130:187881, 2006.

Woodruff, J. M. Peripheral nerve tumors showing glandular differentiation (glandular schwannomas). Cancer, 37:2399413, 1976.

Woodruff, J. M. Pathology of tumors of the peripheral nerve sheath in type 1 neurofibromatosis. Am. J. Med. Genet., 89:23-30, 1999.

Woodruff, J. M.; Chernik, N. L.; Smith, M. C.; Millett, W. B. \& Foote, F. W. Peripheral nerve tumors with rhabdomyosarcomatous differentiation (Malignant "triton" tumors). Cancer, 32:426-39, 1973.

Woodruff, J. M.; Kourea, H. P.; Louis, D. N. \& Scheithauer, B. W. Malignant peripheral nerve sheath tumor (MPNST). In: Kleihues, P.; Cavenee, W. K. (Eds.) Pathology and Genetics of Tumours of the Nervous System. Lyon, France, IARC Press, 2000.

Zhou, H.; Coffin, C. M.; Perkins, S. L.; Tripp, S. R.; Liew, M. \& Viskochil, D. H. Malignant peripheral nerve sheath tumor: a comparison of grade, immunophenotype, and cell cycle/growth activation marker expression in sporadic and neurofibromatosis 1-related lesions. Am. J. Surg. Pathol., 27:1337-45, 2003.

Dirección para correspondencia:

Julio A. Díaz-Pérez

Facultad de Medicina

Universidad de Santander UDES

COLOMBIA

Email: patologia@udes.edu.co

Recibido : 12-10-2010

Aceptado: 11-12-2010 